\section{A STUDY ON RECENT EARTHQUAKES IN AND AROUND BANGLADESH}

\author{
A S M Fahad Hossain*, Nusrat Jahan, Mehedi Ahmed Ansary \\ Department of Civil Engineering, Ahsanullah University of Science \\ and Technology, Dhaka, Bangladesh
}

Article history

Received

27 November 2020

Received in revised form

22 January 2021

Accepted

31 January 2021

Published online

31 March 2021

\section{*Corresponding author \\ fahad.ce@aust.edu}

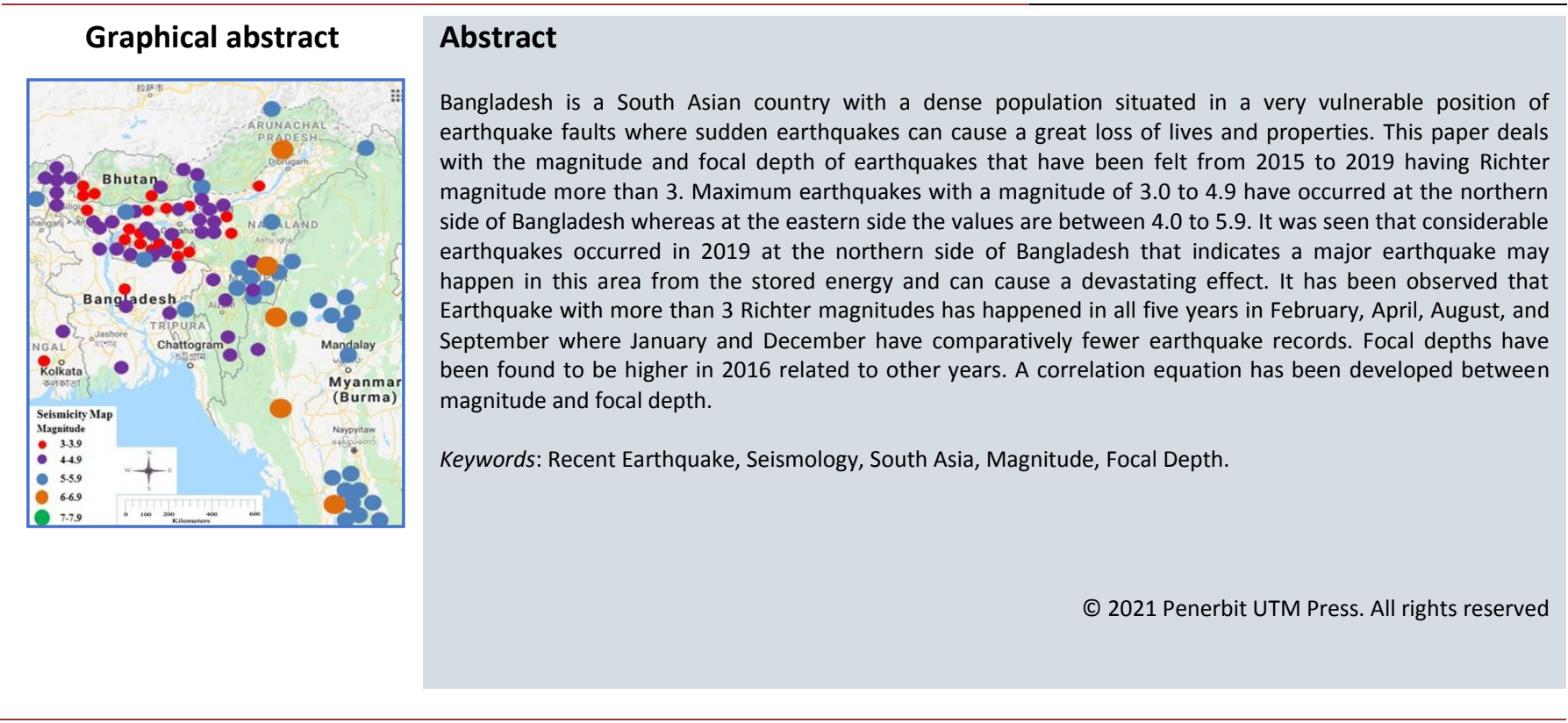

\subsection{INTRODUCTION}

An earthquake is a sudden shaking movement of the earth's surface that is known as a quake, trembled, or tremor. Earthquakes can range in size from those that are so weak that they cannot be felt to those earthquakes violent enough to toss people around and destroy whole cities. The seismic activity of an area refers to the frequency, type, and size of earthquakes experienced over a period of time. Bangladesh is consistently ranked as one of the most vulnerable countries to natural disasters, but this is primarily because of the frequent occurrence of tropical cyclones, floods, heatwaves, and other weather-related disasters, combined with the massive people living in vulnerable conditions. Bangladesh is situated in a modestly dangerous domain and the density of population here and the infrastructural condition are been always a matter of worry here. A recent earthquake in several parts of the universe and the destruction occurred, clearly showing that Bangladesh has to take necessary steps to face the earthquake at any time in the future.

Bangladesh is surrounded mostly by India and a few by Myanmar and she lies where three tectonic plates-Eurasia, India, and Burma meet making her one of the most tectonically active places in the world. Bangladesh has experienced many devastating as well as mild earthquakes in the past 200 years. Bangladesh has been shaken up by more than 250 earthquakes after the independence in 1971and some of those were greater than 6.0 magnitude, but the risks cannot be ignored. The 1897 Great Indian Earthquake with a magnitude of 8.7, which is one of the strongest earthquakes in world history by killing 1542 
and affected almost the whole of Bangladesh (Oldham, 1899). Recently, Bilham et al. (2001) pointed out that, there is a very high possibility that a great earthquake will occur around the Himalayan region based on the difference between energy accumulation in this region and historical earthquake occurrence. The population increase around this region is the last 50 times than the population of 1897 and city like Dhaka has a population exceeding several million. It is a cause for great concern that the next great earthquake may occur in this region at any time. So that detailed seismic response analysis of his area is necessary. Also, observation of recent earthquake records is needed.

\subsection{METHODOLOGY}

\subsection{SEISMOLOGY OF BANGLADESH:}

Bangladesh is located close to the borders of two active plates (Indian plate in the west and Eurasian plate in the east and north) the country has always been under threat of an earthquake that might be so catastrophic by killing people in less than a minute. Calcutta, Assam, Tripura are the three very earthquake-prone regions that are joined to Bangladesh in the boundaries in the northern, western and north-eastern part respectively. If we consider the tectonics and geology, five major faults are significant for the occurrences of devastating earthquakes:
1. Bogra Fault Zone
2. Tripura Fault Zone
3. Shilong Plateau
4. Dauki Fault Zone
5. Assam Fault Zone

Bogra fault is a normal fault and is very close to the Bogra town and Jamuna River that was active in Palaeogene and Neogene times. The movement along the Bogra fault tends to the deposition of a large amount of sedimentary pile within the zone. Tripura is one of the states of India surrounded by Bangladesh and two other states Mizoram and Assam of India, are surrounded by Koplili fault; Kaladan fault etc. that have produced many earthquakes. The Tripura Naga orogenic belt is a zone of highly faulted Tertiary deposits that have witnessed earthquakes of moderate magnitudes and Shillong Plateau is characterized as a seismically active and geologically complex region located on the collision boundary between Indian and Eurasian plate in the Meghalaya state of India. The general altitude of the Plateau is about $1500 \mathrm{~m}$. The plateau is composed of the Precambrian Metamorphic rocks and the Tertiary and Quaternary deposits are limited on the southern foothills of the Shillong Plateau that indicates the successive uplift of the Shillong Plateau and the process started from the Pliocene time. Dauki fault zone is a $300 \mathrm{~km}$ long north dipping reverse fault along the Meghalaya-Bangladesh border and inferred to go through the southern margin of Shillong plateau. It has a major role in deforming the surrounding areas. The Dauki fault is considered to be active in the past. Though it is inactive in recent times still it is considered as one of the major threats for Bangladesh for the occurrence of devastating earthquakes. Figure 1 shows the tectonic framework of Bangladesh.

Dhaka city, the economic, political, cultural, and religious center of Bangladesh is highly vulnerable to the earthquake disaster due to the high density of population, unplanned infrastructure and close proximity with India and Myanmar's active seismic area, poor economic condition, poor emergency preparation, and recovery capability. Bangladesh was divided into four main earthquake zones in BNBC (Bangladesh National Building Code) 2014 where four zones have PGA (Peak Ground Acceleration) values varied to $0.12,0.20,0.28$ and 0.36 . Figure 2 shows the seismic zoning map of Bangladesh according to BNBC 2014. A seismicity map of Bangladeshis is shown in figure 3. 


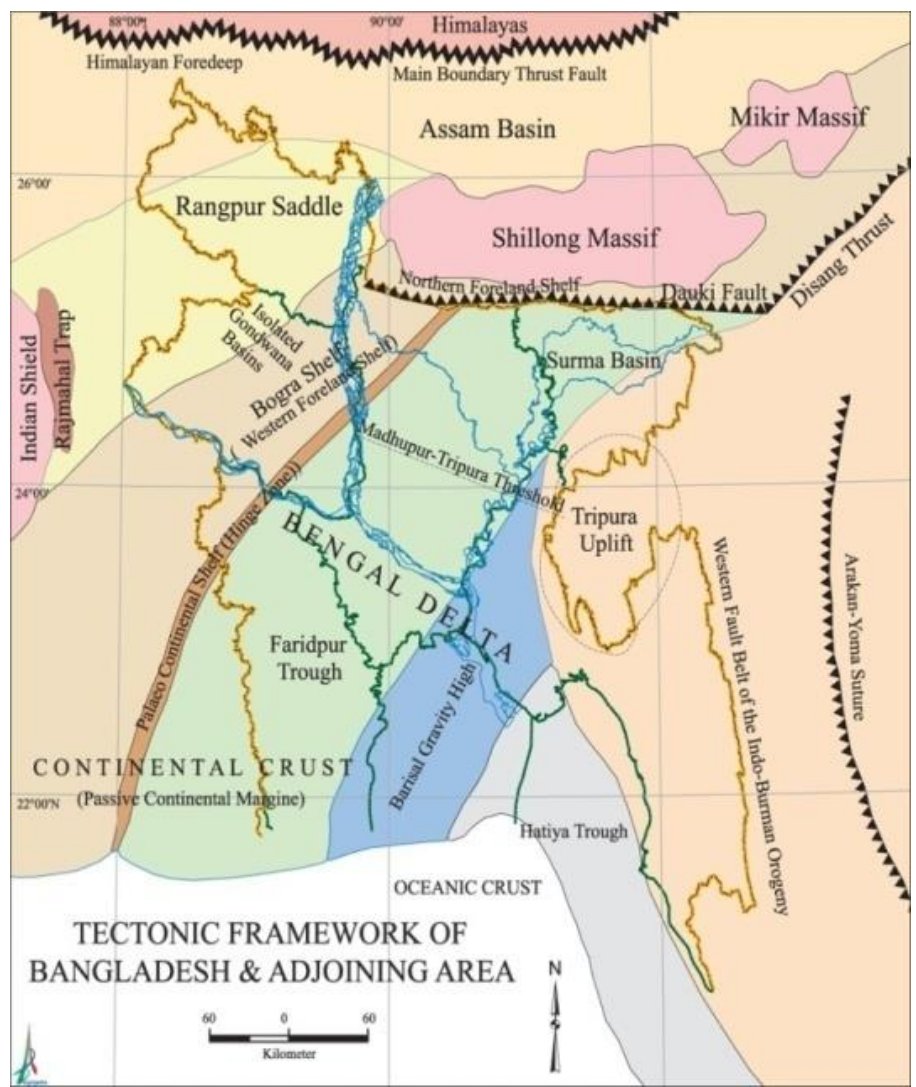

Figure 1 Tectonic Framework of Bangladesh (Banglapedia, 2015)

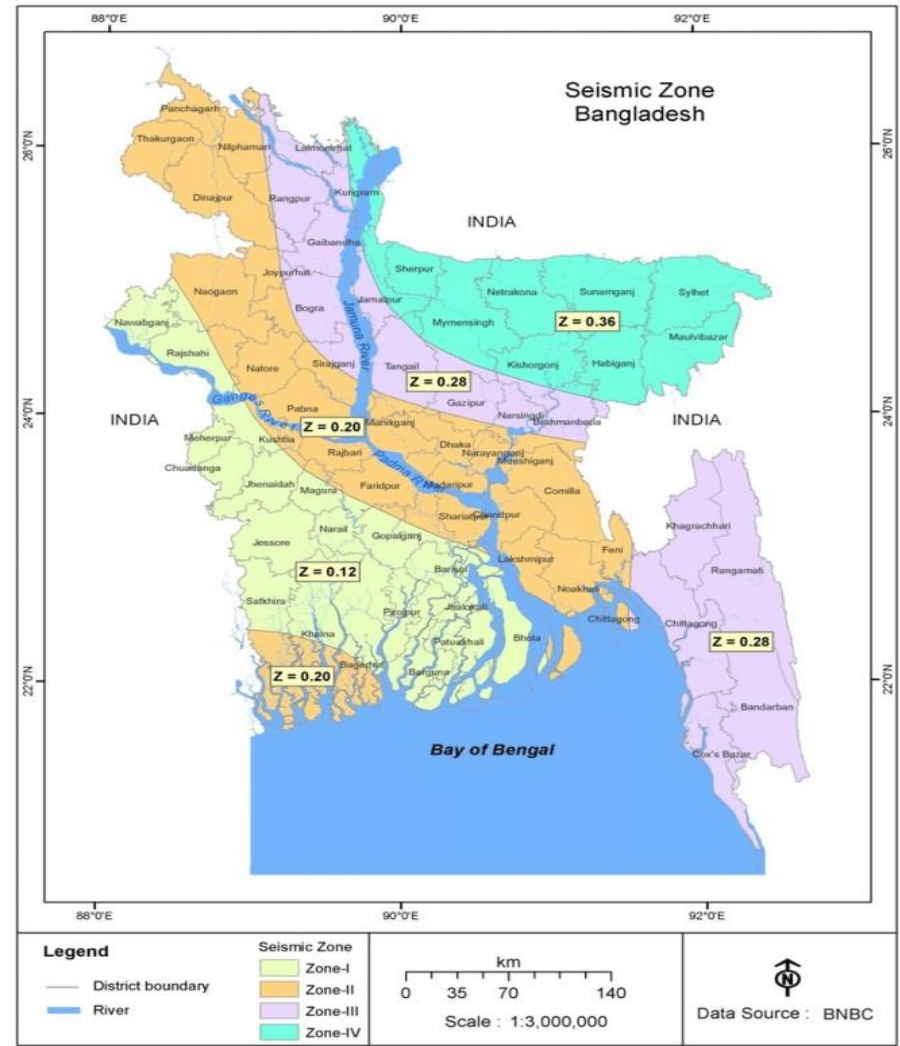

Figure 2: Earthquake zones of Bangladesh (BNBC 2014) 


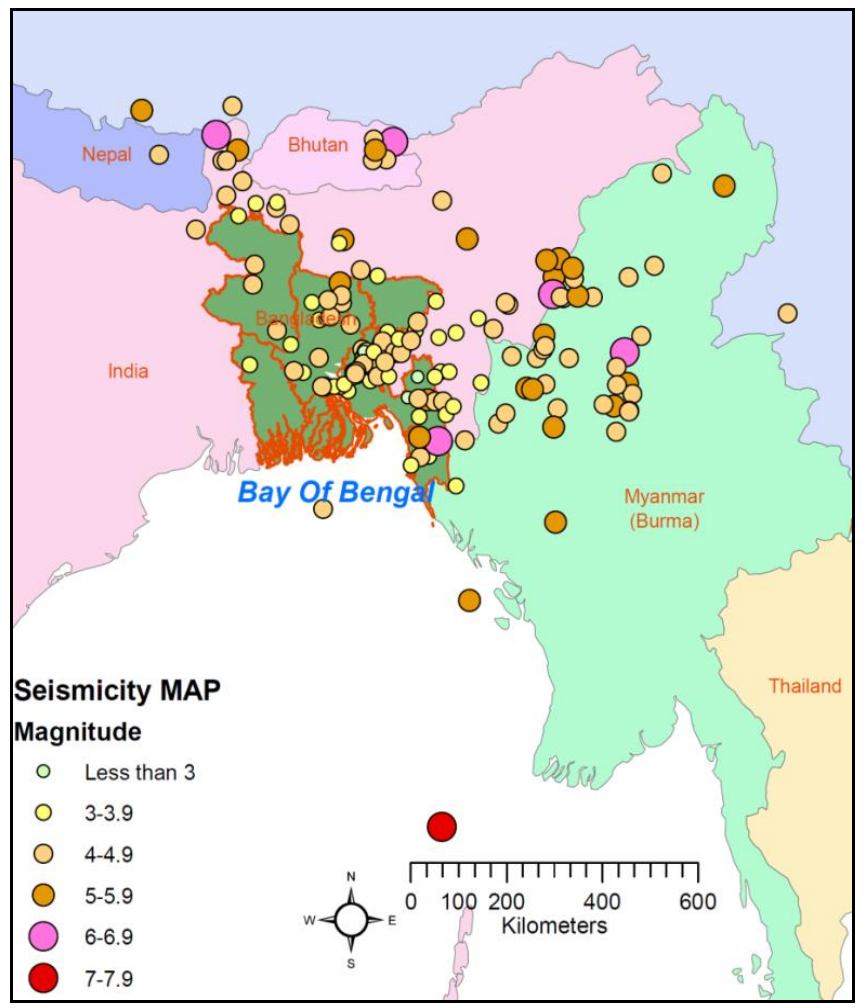

Figure 3: Seismicity Map of Bangladesh (M. A. Ansary, 2018)

\subsection{RESULTS AND DISCUSSION}

\subsection{Recent Earthquake Record (Magnitude And Focal Depth)}

The magnitude, focal depth and locations of five year's earthquake having Richter magnitude more than 3 that was felt from 2015 to 2019 (up to October) near Bangladesh are represented in table 1 to table 5 below.

Table 1: Earthquake Record of 2019 (Up to October)

\begin{tabular}{|c|c|c|c|c|}
\hline \multirow{2}{*}{ Date } & \multirow{2}{*}{ Time } & \multirow{2}{*}{ Earthquake Location } & \multicolumn{2}{|c|}{ Earthquake Parameters } \\
\hline & & & Magnitude & Focal Depth (Km) \\
\hline 07 Oct & 6:35 PM & Mongar, Bhutan & 4.7 & 10 \\
\hline 03 Oct & 12:24 PM & Imphal, Manipur, India & 4.8 & 66.4 \\
\hline 26 Sep & 8:39 PM & East Garo Hills, Meghalaya, India & 3.2 & 5 \\
\hline 23 Sep & 10:27 PM & West Garo Hills, Meghalaya, India & 3.1 & 10 \\
\hline 20 Sep & $3: 40$ AM & East Garo Hills, Meghalaya, India & 3.2 & 5 \\
\hline 09 Sep & $12: 40$ PM & Kashmir- India Border Region & 5.0 & 17 \\
\hline 04 Sep & 2:43 AM & Nadia, West Bengal, India & 4.3 & 33 \\
\hline 31 Aug & 9:09 PM & Shwebo, Sagain, Myanmar & 5.5 & 10 \\
\hline 28 Aug & 7:40 PM & 169 KM NNE of Bamboo Flat, India & 5.0 & 43.1 \\
\hline 18 Aug & 9:24 AM & Pyu, Bago, Myanmar & 5.3 & 40 \\
\hline 06 Aug & 2:08 AM & Dhubri, Assam, India & 3.4 & 33 \\
\hline 04 Aug & $7: 26 \mathrm{AM}$ & Andaman Islands Region, India & 5.1 & 10 \\
\hline 03 Aug & 4:59 PM & Howrah, West Bengal, India & 3.6 & 10 \\
\hline $28 \mathrm{Jul}$ & 4:44 PM & East Garo Hills, Meghalaya, India & 3.6 & 70 \\
\hline $20 \mathrm{Jul}$ & 4:54 AM & Eastern Xizang-India Border Region, India & 5.2 & 10 \\
\hline $19 \mathrm{Jul}$ & $3: 22$ PM & Bomdila, Arunachal Pradesh, India & 5.5 & 16 \\
\hline
\end{tabular}




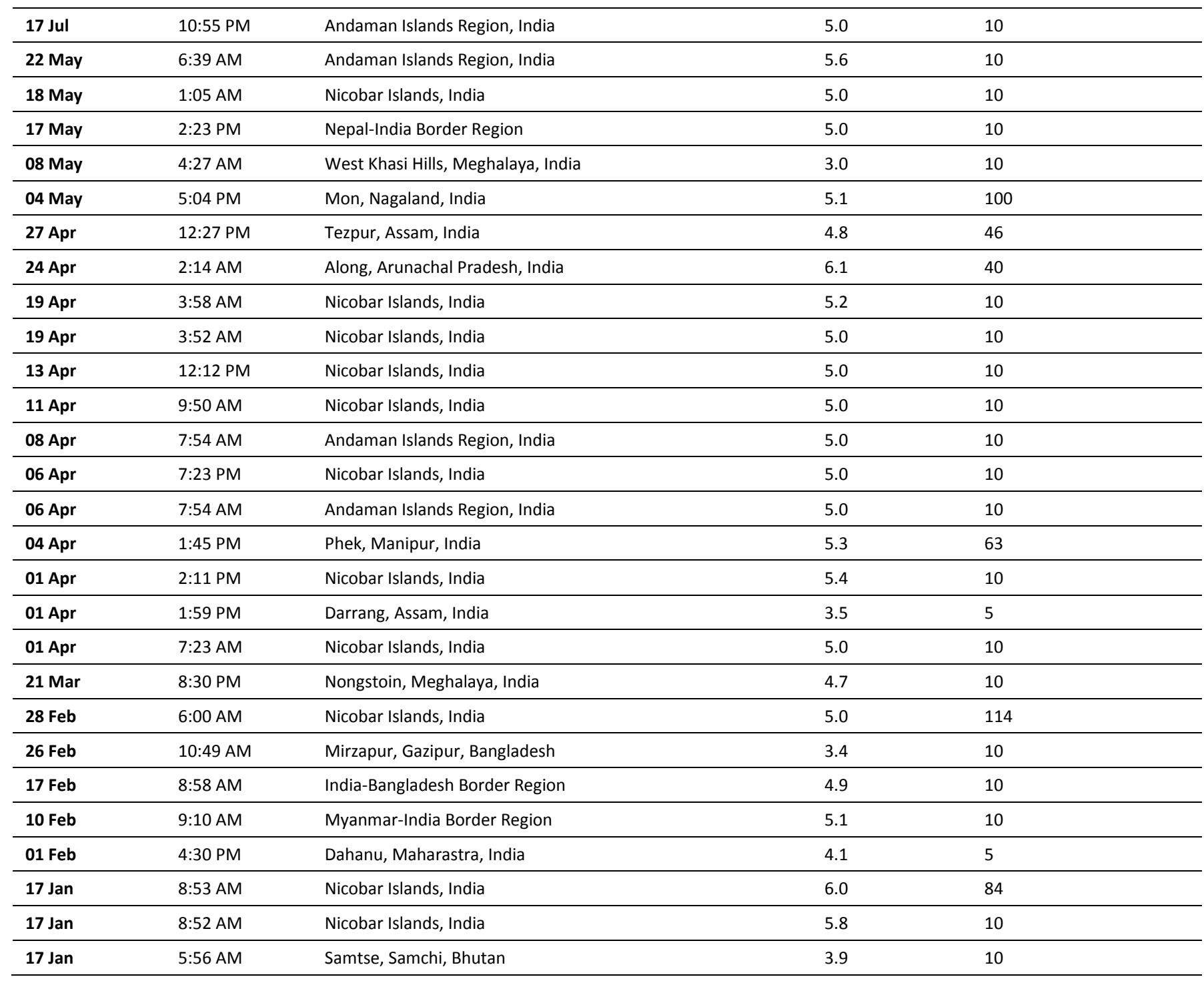

Table 2: Earthquake Record of 2018

\begin{tabular}{|c|c|c|c|c|}
\hline Date & Time & Earthquake Location & \multicolumn{2}{|c|}{ Earthquake Parameters } \\
\hline $28 \mathrm{Nov}$ & 4:17 PM & Myanmar-India Border Region & 5.5 & 10 \\
\hline $01 \mathrm{Dec}$ & 9:07 PM & Tawang, Arunachal Pradesh, India & 4.8 & 10 \\
\hline 29 Nov & 8:43 PM & Kashmir-Xizang Border Region & 5.2 & 8 \\
\hline 10 Oct & 4:28 PM & Pyu, Bago, Myanmar & 5.1 & 10 \\
\hline 01 Oct & 7:24 PM & Andaman Islands, India Region & 5.1 & 38 \\
\hline 25 Sep & 9:47 AM & Barpeta, Assam, India & 4.3 & 10 \\
\hline 14 Sep & 1:51 PM & Nicobar Islands, India Region & 5.2 & 85.6 \\
\hline 11 Aug & 4:04 PM & Panbang, Shemgabg, Bhutan & 3.8 & 10 \\
\hline
\end{tabular}




\begin{tabular}{|c|c|c|c|c|}
\hline 06 Aug & $10: 35 \mathrm{AM}$ & Nicobar Islands, India Region & 5.1 & 10 \\
\hline $22 \mathrm{Jul}$ & 4:04 PM & Off West Coast of Northern Sumatra & 5.2 & 10 \\
\hline $05 \mathrm{Jul}$ & 2:34 AM & Dawel, Tanintharyi, Myanmar & 5.1 & 10 \\
\hline $04 \mathrm{Jul}$ & 1:12 PM & Andaman Islands, India Region & 5.2 & 10 \\
\hline 17 Jun & 9:07 PM & Mangan, Sikkim, India & 4.4 & 50 \\
\hline 17 Jun & 7:21 PM & Pyu, Bago, Myanmar & 5.0 & 10 \\
\hline 11 Jun & $10.53 \mathrm{AM}$ & Dhing, Assam, India & 4.9 & 10 \\
\hline 28 May & $8: 00 \mathrm{AM}$ & Nicobar Islands, India Region & 5.0 & 101 \\
\hline 14 May & $12: 20 \mathrm{AM}$ & Nicobar Islands, India Region & 5.0 & 10 \\
\hline 24 Apr & 6:33 PM & Pyu, Bago, Myanmar & 5.1 & 10 \\
\hline 24 Apr & 10:08 AM & Mawlaik, Sagain, Myanmar & 5.2 & 105 \\
\hline 21 Apr & 4:29 AM & Pyu, Bago, Myanmar & 5.0 & 17 \\
\hline 11 Apr & $5: 45$ AM & Singrauli, India & 4.6 & 10 \\
\hline $18 \mathrm{Mar}$ & 1:59 AM & Pyu, Bago, Myanmar & 5.1 & 19 \\
\hline 09 Mar & $3: 13$ AM & Nay Pyl Taw, Mandalay, Myanmar & 5.3 & 10 \\
\hline 13 Feb & 8:39 AM & Andaman Islands, India Region & 5.5 & 10 \\
\hline 29 Jan & 3:02 AM & Meghalaya, India & 3.4 & 10 \\
\hline 25 Jan & 9:48 AM & Nicobar Islands, India Region & 5.1 & 10 \\
\hline 25 Jan & 7:15 AM & Nicobar Islands, India Region & 5.7 & 10 \\
\hline 20 Jan & 7:14 AM & India-Bangladesh Border Region & 4.6 & 10 \\
\hline 12 Jan & $12: 44 \mathrm{AM}$ & Pyu, Bago, Myanmar & 5.2 & 10 \\
\hline 12 Jan & $12: 42 \mathrm{AM}$ & Pyu, Bago, Myanmar & 5.2 & 10 \\
\hline 12 Jan & $12: 38 \mathrm{AM}$ & Pyu, Bago, Myanmar & 5.3 & 10 \\
\hline 12 Jan & $12: 26 \mathrm{AM}$ & Pyu, Bago, Myanmar & 6.0 & 10 \\
\hline 07 Jan & 12:47 PM & Wangjing, Manipur, India & 5.5 & 22 \\
\hline 06 Jan & $2: 23 \mathrm{AM}$ & Assam, India & 3.4 & 10 \\
\hline 03 Jan & 2:47 AM & Nicobar Islands, India Region & 5.1 & 13 \\
\hline
\end{tabular}

Table 3: Earthquake Record of 2017

\begin{tabular}{|c|c|c|c|c|}
\hline \multirow{2}{*}{ Date } & \multirow{2}{*}{ Time } & \multirow{2}{*}{ Earthquake Location } & \multicolumn{2}{|c|}{ Earthquake Parameters } \\
\hline & & & Magnitude & Focal Depth (Km) \\
\hline $29 \mathrm{Dec}$ & 5:29 PM & Nicobar Islands, India Region & 5.2 & 10 \\
\hline $29 \mathrm{Dec}$ & $2: 23 \mathrm{AM}$ & Monywa, Sagain, Myanmar & 5.0 & 86 \\
\hline $22 \mathrm{Dec}$ & 9:58 PM & Andaman Islands, India Region & 5.1 & 10 \\
\hline $11 \mathrm{Dec}$ & $9: 35 \mathrm{AM}$ & Nongstoin, Meghalaya, India & 4.7 & 10 \\
\hline $08 \mathrm{Dec}$ & 11:55 PM & Meghalaya, India & 3.0 & 10 \\
\hline $06 \mathrm{Dec}$ & 9:19 PM & Western-Xizang Border region, India & 5.0 & 10 \\
\hline $03 \mathrm{Dec}$ & $5: 43$ AM & Naya Bazar, Sikkim, India & 4.2 & 10 \\
\hline 08 Nov & 10:50 AM & India-Bangladesh Border Region & 4.7 & 10 \\
\hline 07 Sep & 8:32 PM & Tura, Meghalaya, India & 4.4 & 10 \\
\hline 06 Sep & $6: 10$ AM & Andaman Islands, India Region & 5.4 & 10 \\
\hline 03 Sep & $12: 52$ PM & Nicobar Islands, India Region & 5.0 & 10 \\
\hline 03 Aug & $12: 18$ AM & Churachandpur, Manipur, India & 5.2 & 53 \\
\hline 05 Jun & $10: 40$ PM & India-Bangladesh Border Region & 3.0 & 96 \\
\hline 05 May & 11:58 PM & Andaman Islands, India Region & 5.0 & 10 \\
\hline
\end{tabular}




\begin{tabular}{|c|c|c|c|c|}
\hline $18 \mathrm{Apr}$ & 8:01 PM & Meghalaya, India & 4.3 & 10 \\
\hline $18 \mathrm{Apr}$ & 11:11 AM & Kashmir-India Border Region & 5.1 & 10 \\
\hline 16 Apr & 2:08 PM & Assam, India & 3.3 & 24 \\
\hline $30 \mathrm{Mar}$ & 1:14 AM & Assam, India & 3.5 & 18 \\
\hline 27 Mar & $3: 42 \mathrm{AM}$ & Gangtok, Sikkim, India & 4.5 & 10 \\
\hline 14 Mar & 8:51 AM & Nicobar Islands, India Region & 5.9 & 10 \\
\hline 13 Mar & 8:19 PM & Tharrawaddy, Bago, Myanmar & 5.1 & 10 \\
\hline 07 Mar & $4: 28$ PM & Samtse, Samchi, Bhutan & 3.9 & 10 \\
\hline 04 Mar & $8: 11 \mathrm{AM}$ & Phek, Manipur, India & 5.1 & 75 \\
\hline 20 Jan & $1: 18 \mathrm{AM}$ & Tawang, Arunachal Pradesh, India & 4.1 & 10 \\
\hline 04 Jan & 12:49 AM & Mawlaik, Sagain, Myanmar & 5.0 & 110 \\
\hline 03 Jan & 3:09 PM & Ambassa, Tripura, India & 5.7 & 32 \\
\hline
\end{tabular}

Table 4: Earthquake Record of 2016

\begin{tabular}{|c|c|c|c|c|}
\hline \multirow[t]{2}{*}{ Date } & \multirow[t]{2}{*}{ Time } & \multirow[t]{2}{*}{ Earthquake Location } & \multicolumn{2}{|c|}{ Earthquake Parameters } \\
\hline & & & Magnitude & Focal Depth (Km) \\
\hline $01 \mathrm{Dec}$ & 1052 PM & Nepal-India Border Region & 5.0 & 18 \\
\hline 28 Nov & $5: 35 \mathrm{AM}$ & Namche Bazar, Nepal & 5.4 & 10 \\
\hline 23 Oct & 9:56 PM & India-Bangladesh Border Region & 4.6 & 28 \\
\hline 19 Oct & 1:20 PM & India-Bangladesh Border Region & 4.4 & 36 \\
\hline 15 Sep & $8: 30 \mathrm{AM}$ & Andaman Islands, India Region & 5.1 & 10 \\
\hline 24 Aug & $4: 34$ PM & Chauk, Magway, Myanmar & 6.8 & 84 \\
\hline 23 Aug & $8: 11 \mathrm{AM}$ & Mawlaik, Sagain, Myanmar & 5.3 & 106 \\
\hline 11 Aug & 4:55 PM & Nicobar Islands, India Region & 5.2 & 10 \\
\hline 27 Jun & $6: 27 \mathrm{AM}$ & India-Bangladesh Border Region & 4.7 & 10 \\
\hline 12 May & 1:54 PM & Myanmar-India Border Region & 5.3 & 10 \\
\hline 09 May & 10:03 PM & Andaman Islands, India Region & 5.3 & 39 \\
\hline $20 \mathrm{Apr}$ & $2: 32 \mathrm{AM}$ & Andaman Islands, India Region & 5.0 & 10 \\
\hline 19 Apr & $11: 22$ PM & Mangan, Sikkim, India & 4.3 & 60 \\
\hline $13 \mathrm{Apr}$ & 7:55 PM & Mawlaik, Sagain, Myanmar & 6.9 & 134 \\
\hline 05 Apr & $1: 42 \mathrm{PM}$ & India-Bangladesh Border Region & 5.0 & 18 \\
\hline 13 Mar & $4: 30 \mathrm{PM}$ & Marigaon, Assam, India & 4.3 & 58 \\
\hline 12 Mar & $10: 15$ PM & Birpara, West Bengal, India & 3.9 & 14 \\
\hline $22 \mathrm{Feb}$ & $12.10 \mathrm{AM}$ & Nepal-India Border Region & 5.1 & 11 \\
\hline $21 \mathrm{Feb}$ & 12:09 PM & Nepal & 5.5 & 10 \\
\hline $14 \mathrm{Feb}$ & $3: 37$ AM & Samtse, Samchi, Bhutan & 3.7 & 46 \\
\hline $05 \mathrm{Feb}$ & $10: 20 \mathrm{PM}$ & Kathmandu, Nepal & 5.2 & 10 \\
\hline 04 Jan & 5:05 AM & Imphal, Manipur, India & 6.7 & 55 \\
\hline
\end{tabular}


Table 5: Earthquake Record of 2015

\begin{tabular}{|c|c|c|c|c|}
\hline \multirow{2}{*}{ Date } & \multirow{2}{*}{ Time } & \multirow{2}{*}{ Earthquake Location } & \multicolumn{2}{|c|}{ Earthquake Parameters } \\
\hline & & & Magnitude & Focal Depth $(\mathrm{Km})$ \\
\hline $23 \mathrm{Dec}$ & $3: 22 \mathrm{PM}$ & $\underline{\text { Hojai }}, \underline{\text { Assam }}$ India & 4.3 & 48 \\
\hline $22 \mathrm{Dec}$ & $5: 52 \mathrm{AM}$ & Gafargaon, Dhaka, Bangladesh & 4.0 & 14 \\
\hline 30 Oct & 4:15 AM & $\underline{\text { Hailakandi, Assam, India }}$ & 4.8 & 36 \\
\hline 29 Oct & $5: 54 \mathrm{AM}$ & Darlawn, Mizoram, India & 4.3 & 38 \\
\hline 10 Oct & $7: 48 \mathrm{AM}$ & $\underline{\text { Gangtok, Sikkim, India }}$ & 4.3 & 48 \\
\hline 02 Oct & $5: 29 \mathrm{PM}$ & $\underline{\text { Hakha, }}$ Chin, Myanmar & 4.6 & 42 \\
\hline 25 Sep & 10:57 PM & $\underline{\text { Rangia, }}$ Assam, India & 4.4 & 48 \\
\hline 12 Sep & $5: 56 \mathrm{AM}$ & $\underline{\text { Dinhata, }}$ West Bengal, India & 4.0 & 18 \\
\hline 05 Sep & 11:39 AM & $\underline{\text { Hojai, Assam, India }}$ & 4.2 & 47 \\
\hline 29 Aug & 1:21 AM & 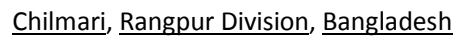 & 4.2 & 13 \\
\hline 21 Aug & 1:21 AM & $\underline{\text { Lakhipur, Assam, India }}$ & 3.9 & 42 \\
\hline $10 \mathrm{Jul}$ & 6:53 PM & Bongaigaon, Assam, India & 4.3 & 19 \\
\hline 30 Jun & 7:15 PM & $\underline{\text { Goalpara, }}$ Assam, India & 4.0 & 42 \\
\hline 28 Jun & 7:05 PM & Basugaon, Assam, India & 5.1 & 26 \\
\hline 25 May & 9:59 PM & Saiha, Mizoram, India & 4.5 & 53 \\
\hline 16 May & 5:34 PM & Kothari, Nepal & 5.5 & 7 \\
\hline 12 May & 1:05 PM & $\underline{\text { Kothari, Nepal }}$ & 7.3 & 15 \\
\hline 12 May & 1:36 PM & $\underline{\text { Kothari, Nepal }}$ & 6.3 & 15 \\
\hline 12 May & 1:17 PM & Kothari, Nepal & 5.5 & 13 \\
\hline $08 \mathrm{Apr}$ & 7:43 AM & $\underline{\text { Sarankhola, Khulna, Bangladesh }}$ & 4.6 & 17 \\
\hline $27 \mathrm{Apr}$ & 6:35 PM & $\underline{\text { Ilam, Eastern Region, Nepal }}$ & 5.1 & 31 \\
\hline $26 \mathrm{Apr}$ & 1:09 PM & $\underline{\text { Kothari, Nepal }}$ & 6.7 & 22 \\
\hline $25 \mathrm{Apr}$ & 3:17 PM & Lobuche, Eastern Region, Nepal & 5.7 & 10 \\
\hline $25 \mathrm{Apr}$ & 12:56 PM & Kothari, Nepal & 5.5 & 10 \\
\hline $25 \mathrm{Apr}$ & 12:18 PM & Kothari, Nepal & 5.6 & 10 \\
\hline $25 \mathrm{Apr}$ & 12:11 PM & Bharatpur, Central Region, Nepal & 7.8 & 8 \\
\hline 15 Jan & 7:33 AM & $\underline{\text { Lunglei, }}$ Mizoram, India & 4.3 & 50 \\
\hline 05 Jan & 9:39 PM & Churachandpur, Manipur, India & 4.2 & 61 \\
\hline
\end{tabular}

\subsection{Graphical Representation Of Earthquake Record According To Magnitude And Year}

From the earthquake records of five years shown above, two maps were prepared according to magnitude and earthquake occurrence year. They are shown in figure 4 and figure 5 . From the magnitude graph, it was seen that earthquake with a magnitude of 5 to 7.9 and 3 to 4.9 occurred at Nepal and northern side of Bangladesh (the area between Bangladesh and Bhutan) respectively. At the eastern side of Bangladesh (the portion between Bangladesh and Myanmar), the values were within 4 to 5.9. From the year wise graph, it was seen that considerable earthquake records were at the northern outside of Bangladesh in the last year 2019 that indicates a major earthquake may happen here from the stored energy and can make a devastating effect. 


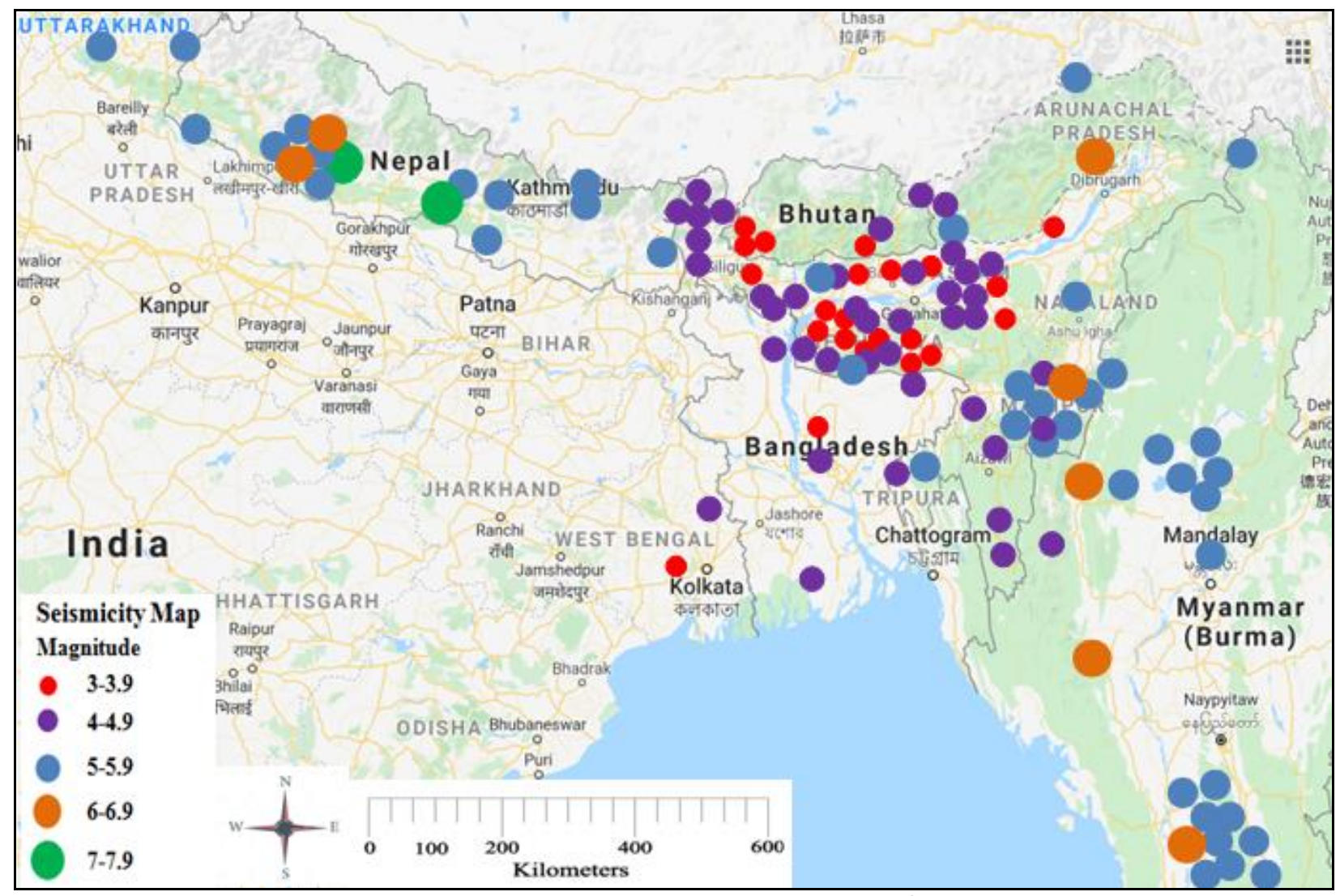

Figure 4: Earthquake Magnitude in and around Bangladesh from 2015 to 2019

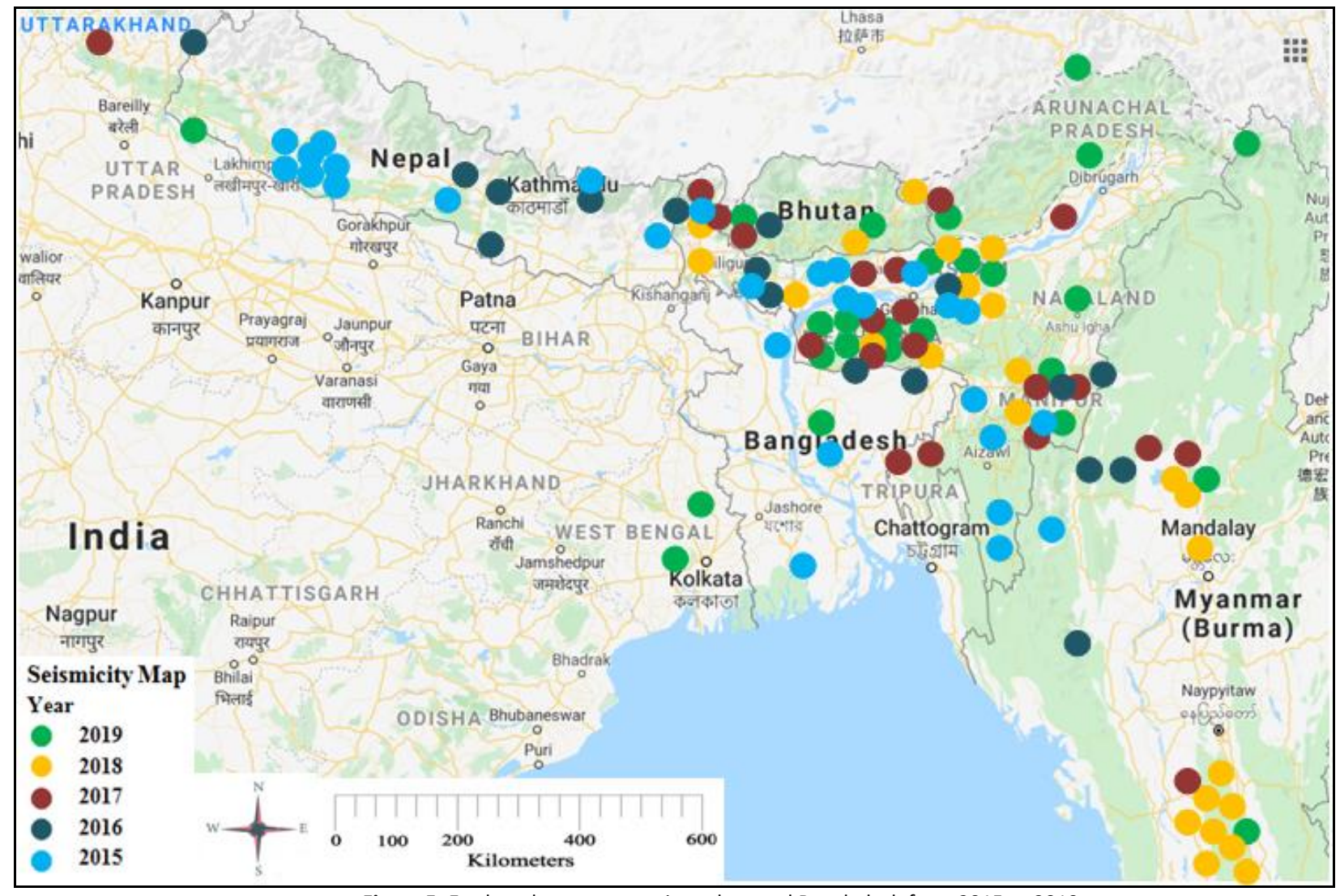

Figure 5: Earthquake occurrence in and around Bangladesh from 2015 to 2019 


\subsection{Comparison between Magnitude and Focal Depth of Different Earthquake in Various Dates:}

A comparison between magnitude and focal depth of different earthquake in 2019 (up to October), 2018, 2017, 2016, and 2015 are shown in figure 6 and figure 7. It was seen that
Earthquake with more than 3 Richter magnitudes occurred in all five years in February, April, August, and September. January and December have comparatively less earthquake records. Focal depth was higher in 2016 related to others in April of August.

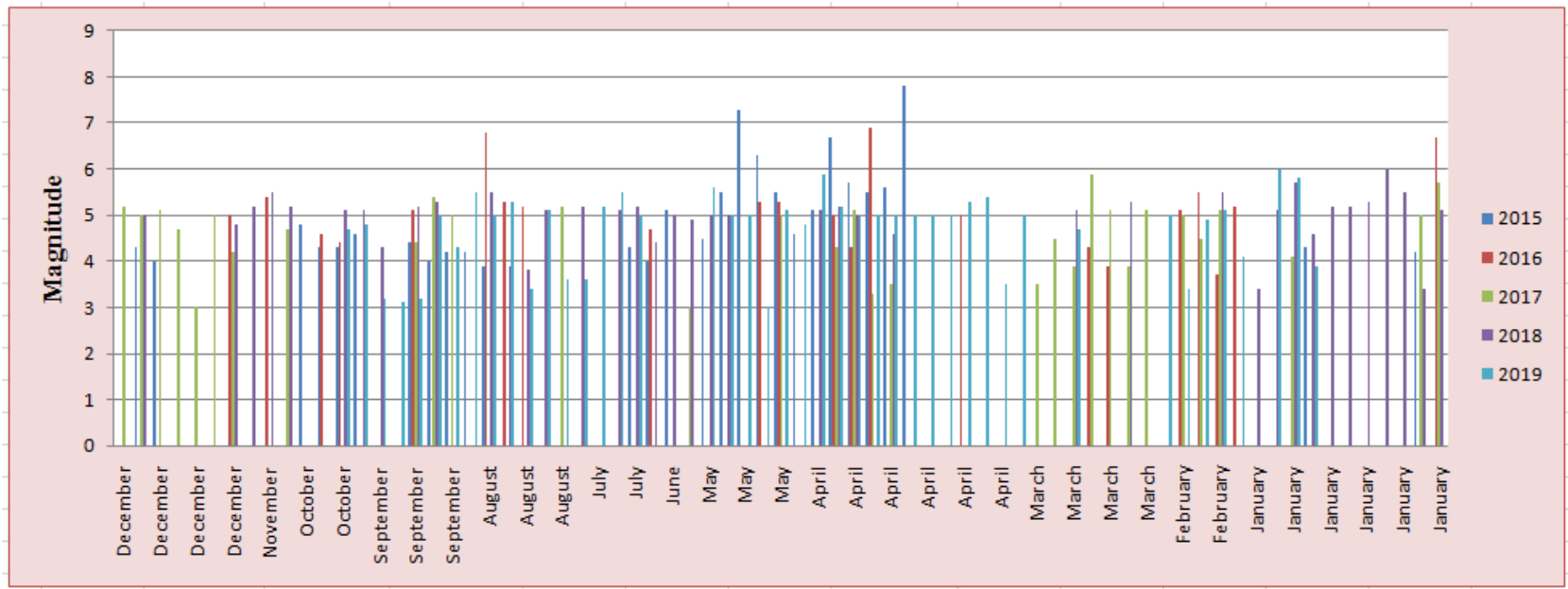

Figure 6: Comparison between magnitudes of different earthquake from 2015 to 2019

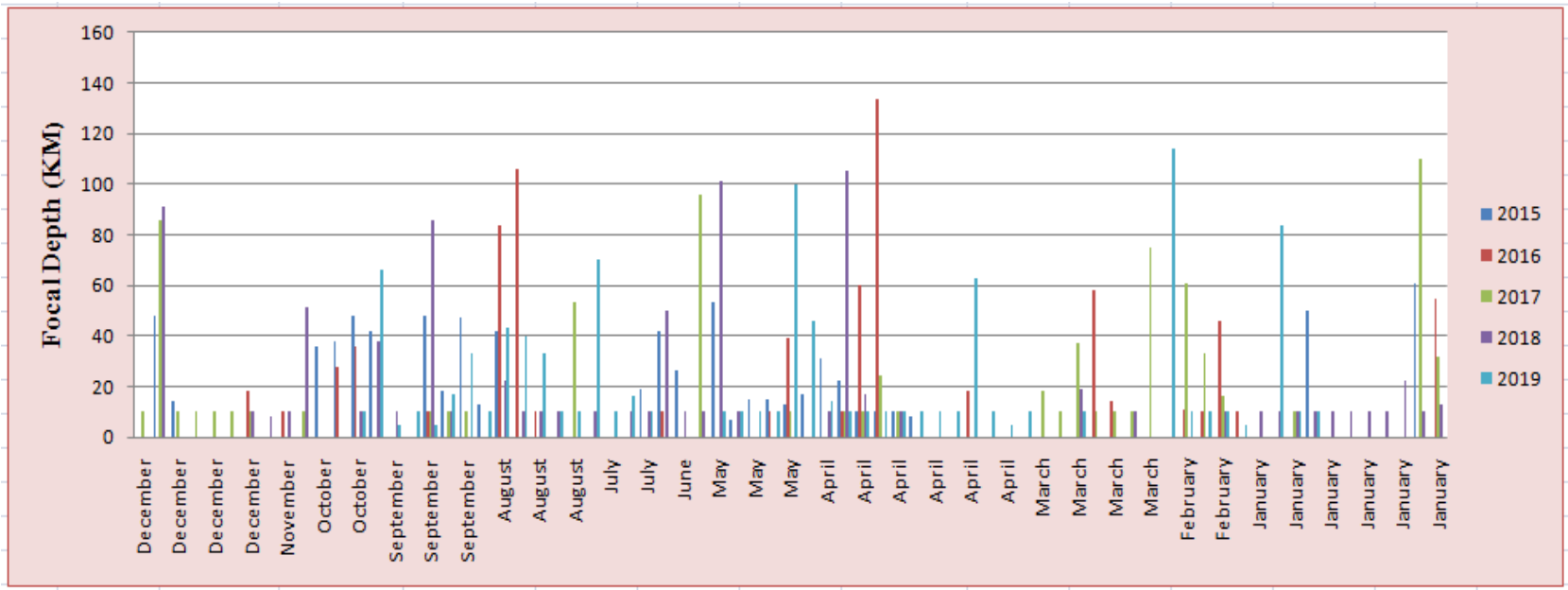

Figure 7: Comparison between focal depths of different earthquake from 2015 to 2019

All data of magnitude vs focal depth of earthquake records shown in this article were plotted in a graph and an equation was developed from the straight-line graph. The straight-line graph is shown in figure 8 and the equation is given below.

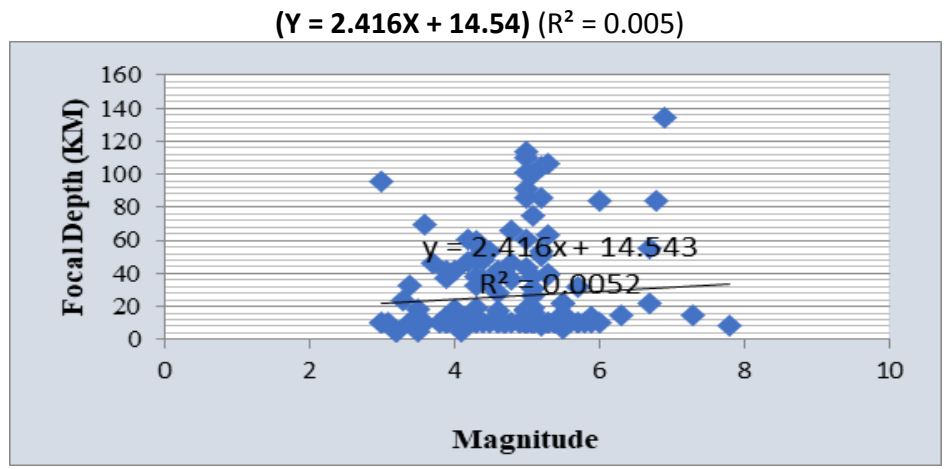

Figure 8: Focal Depth vs Magnitude of different earthquakes from 2015 to 2019 


\subsection{CONCLUSIONS}

In this review article, only earthquake records were demonstrated and earthquake occurring zones and frequency of earthquake were simply analyzed and described. But earthquake hazard analysis of different portions of that earthquakes should be analyzed. The topographical position and the historical quakes obviously demonstrate that the administration of Bangladesh should put much stress on seismic tremors and how to limit its harm. The disaster management system of Bangladesh should be more developed to minimize the loss during the earthquake. The building codes should be followed by the citizens and the government should try to increase the awareness about the safety rules of earthquakes among the mass people.

\section{References}

[1] Alam E 2017 A historical and geographical analysis of earthquake and associated tsunami events on Bangladesh Coast. Journal of Geography $12:$ 54-60. https://doi.org/10.9734/JGEESI/2017/31187

[2] Saha MK 2010 Earthquake: Emerging threat for Dhaka city. Nature Geoscience 6: 15.

[3] Bilham R 2004 Earthquakes in India and the Himalaya: tectonics, geodesy and history. Annals of Geophysics 47: 2-3. https://doi.org/10.4401/ag-3338

[4] Ahmed MNQ 2007 Public awareness on earthquake: A study on a residential area under Sylhet City of Bangladesh. Young 18: 36.

[5] M. A. Ansary, M. R. Arefin and Ripon Hore. 2018. "Development of liquefaction potential map of Dhaka city using SPT test" Journal of Civil Engineering (IEB). 46: 2127-140.

[6] Hossain A.S.M. F., Ansary M. A., Bari Q. H., Adhikari T. L. 2016 Consequence_of_Nepal_Earthquake_2015_and_Effects_in_Bang ladesh; 3rd International Conference on Civil Engineering for Sustainable Development (ICCESD 2016).

[7] Hossain A.S.M. F., Ansary M. A., Bari Q. H., Adhikari T. L. 2016 Consequence_of_Nepal_Earthquake_2015: A Review; Geotechnical Engineering Journal of the SEAGS \& AGSSEA. 46(4): 114-120. December 2015 ISSN 0046-5828

[8] Al-Zaman MDA, Monira NJ 2017 A study of earthquakes in bangladesh and the data analysis of the earthquakes that were generated in Bangladesh and its' very close regions for the last forty. Journal of Geology \& Geophysics. 6(4): 300.

[9] Hossain, A S M Fahad; Islam, N. and Ansary, M. A. 2015 "Use of PS Logging and Ground Response Analysis using DEEPSOIL in BUET-JIDPUS, BUET, Dhaka" International Journal of Innovative Science and Modern Engineering (IJISME) 3(3), (February 2015). 8-12. ISSN: 2319-6386,

[10] Hossain, A S M Fahad; Ansary, M. A. 2015. "Shear Wave Velocity as a Function of Standard Penetration Number and Depth in Dhaka City, Bangladesh" IOSR Journal of Mechanical and Civil Engineering 12(3) Ver. II (May. -Jun. 2015): 31-37. e-ISSN: 22781684, p-ISSN: 2320-334X,

[11] Hossain, A S M Fahad; Ansary, M. A. 2016 "Ps Logging for Site Response Analysis in Dhaka City" in The AUST Journal of Science and Technology 6(1\&2): 83-99. January 2014 and July 2014 (Published in May 2017). (ISSN 2072-0149)

[12] Islam, N.; Alam M. J.; Hossain, A S M Fahad. 2015 "One dimensional Ground Response Analysis for soil in Dhaka and
Khulna Region" CICM 2015, First International Conference on Advances in Civil Infrastructure and Construction Materials, MIST, Dhaka, Bangladesh, 14-15 December 2015.

[13] Javed, S. A.; Sameer M.; Hossain, A S M Fahad and Helaly, A. L. 2016. "Ground Investigation and Response of Jhilmil Residential Town Project" $3^{\text {rd }}$ International Conference on Advances in Civil Engineering 2016 (ICACE 2016) 21 -23 December 2016 CUET, Chittagong, Bangladesh

[14] Raqib Al Mahmood, A. S. M. Fahad Hossain, \& Md. Munirul Islam. 2020. A Comparison between Linear and Equivalent Linear Ground Response Analysis of the Sub-Soil of Ganakbari, Dhaka, Bangladesh. Journal of Earthquake Science and Soil Dynamics Engineering. 3(1): 1-13. http://doi.org/10.5281/zenodo.3822014

[15] Banglapedia Contributors. 2015. Tectonic Framework; Banglapedia, National Encyclopedia of Bangladesh. Retrieved 06:52, March21, 2021, from (http://en.banglapedia.org/index.php?title=Tectonic_Framewor)

[16] Wikipedia contributors. 2020. Geology of Bangladesh. In Wikipedia, The Free Encyclopedia. Retrieved 06:52, March 21, 2021,fromhttps://en.wikipedia.org/w/index.php?title=Geology_ of Bangladesh\&oldid $=971078778$

[17] Banglapedia Contributors. 2015. Seismic Zone; Banglapedia, National Encyclopedia of Bangladesh. Retrieved 13:12, March 21, 2021, from (http://en.banglapedia.org/index.php?title=Seismic_Zone).

[18] Volcano Discovery Contributors. 2021. Latest earthquakes in or near Asia, past 30 days; Volcano Discovery. Retrieved 14:15, March 21, 2021, from (https://www.volcanodiscovery.com/earthquakes/asia.html).

[19] Volcano Discovery Contributors. 2021. Latest Earthquakes in Bangladesh; Volcano Discovery. Retrieved 14:11, March 21, 2021,

from (https://www.volcanodiscovery.com/earthquakes/bangladesh.ht $\mathrm{ml}$ )

[20] Wikipedia contributors. 2020. 2017 Tripura earthquake. In Wikipedia, The Free Encyclopedia. Retrieved 07:44, March 21 2021,

from https://en.wikipedia.org/w/index.php?title=2017_Tripura_earth quake\&oldid $=964711044$

[21] Wikipedia contributors. 2021. United States Geological Survey. In Wikipedia, The Free Encyclopedia. Retrieved 07:43, March 21 2021, from https://en.wikipedia.org/w/index.php?title=United_States_Geol ogical_Survey\&oldid=1011628007

[22] Latest Earthquakes. [Online]. Available: https://earthquake.usgs.gov/earthquakes/map/. [Accessed: 21Mar-2021]

[23] Wikipedia contributors. 2021. April 2015 Nepal earthquake. In Wikipedia, The Free Encyclopedia. Retrieved 07:31, March 21, 2021, from https://en.wikipedia.org/w/index.php?title=April_2015_Nepal_e arthquake\&oldid $=1012750693$

[24] Banglapedia Contributors. 2015. Earthquake; Banglapedia, National Encyclopedia of Bangladesh. Retrieved 13:20, March 21 2021 (http://en.banglapedia.org/index.php?title=Earthquake)

[25] Columbia University. 2016. A giant quake may lurk under Bangladesh and beyond. Retrieved 21 March 2021 from https://phys.org/news/2016-07-giant-quake-lurk bangladesh.html 